\title{
Raz Chen-Morris
}

Measuring Shadows: Kepler's Optics of Invisibility (University Park, PA:

Pennsylvania State University Press, 2016), pp. 264, ills., \$79.95, ISBN 9780271 070988.

Johannes Kepler's 1604 optical treatise Ad Vitellionem represents an important moment in early modern optics. Boldly claiming that the nature of light was essential to a theory of optics, while at the same time introducing the inverted retinal image, Kepler's treatise has been accorded a prominent position within the historiography of optics. The novelty that Raz Chen-Morris provides in Measuring Shadows: Kepler's Optics of Invisibility is the statement that the methodological richness of Kepler's optics is truncated without due attention to Kepler's own intellectual development and context. In particular, as ChenMorris contends, Kepler's Ad Vitellionem should be seen as addressing the visual problems raised by the publication of Nicolaus Copernicus's $D e$ revolutionibus in 1543 . One important implication of Copernicus's treatise was that it replaced daily sense-experience with an invisible motion of the earth, dislodging perception as the basis of human knowledge. Chen-Morris's book explains how Kepler's optical treatise, the title of which self-identifies as 'the optical part of astronomy, reorganized the discipline of optics in light of Copernican astronomy. Kepler's aim was an a priori astronomy, and his work in optics was one important step along the way.

Consisting of six chapters, an Introduction and a brief Conclusion, the book exhibits an internal organization that may be understood as falling in two distinct parts. The first part, chapters 1-3, covers the methodological shifts that Kepler introduced. Chen-Morris explains how Kepler reorganized the medieval perspectiva, which included the camera obscura and pinhole analysis, to create an epistemology that could respond to Copernican astronomy. In its place Kepler developed a framework in which light, as interpreted by its shadows, was the agency of vision and could be understood mathematically. To establish this aspect, Kepler overcame the traditional Aristotelian identification of mathematics as mere abstraction from the physical world by utilizing the pinhole analysis to demonstrate that mathematical entities were representations of physical motion. These ideas, which were based upon observations not available to the naked eye, are, for Chen-Morris, the way Kepler established an optics of invisibility.

The second part of the book, chapters 4 to 6 , situates the significance of Kepler's optical epistemology within larger cultural and intellectual frameworks, namely Renaissance art, the emblematic tradition as evidenced by Robert Fludd, and seventeenth-century astronomy. Despite Kepler's affinities 
with the artistic tradition - particularly given that, according to Kepler, vision occurred as a result of a 'picture' on the retina - Chen-Morris nevertheless distinguishes the role of the geometric ideal within Renaissance art from Kepler's acceptance of the non-ideal geometric pattern, such as in his theory of elliptical orbits. According to Chen-Morris, Renaissance artists idealized the world, thereby creating a human construct, whereas Kepler focused on the recognizable geometric patterns that could be observed. A similar analysis, that of the distinction between human construction and geometric pattern, is behind Chen-Morris's interpretation of the distinction between Robert Fludd's emblematic understanding of the natural world and Kepler's geometrical understanding.

Many of these interpretive threads are brought together once more in the final chapter of the book, where Chen-Morris considers the significance of Kepler's optics of invisibility within Kepler's larger astronomical interests. It is here that Chen-Morris's insights shine the most, as he encourages the historian to interpret Kepler's optics not only in relation to optical texts, but as Kepler himself intended, in connection to astronomy. As Chen-Morris indicates, in Kepler's later works, such as the Strena and Somnium, many of the optical, philosophical, and mathematical theories that he had previously developed in his Ad Vitellionem are utilized. It is for this insight and contextualization that this book will be of interest to historians of optics, astronomy, mathematics, and philosophy. While the book does pass quickly over certain complex topics without exhaustive analyses, its greatest strength is its comprehensive approach. It will be difficult to find as helpful an introduction to Kepler's optics as the book Chen-Morris has produced.

In addition to the more narrow scope of Kepler's optics, the reader of Chen-Morris's work will be delighted to find that he weaves together many interesting cultural allusions that pertain to the broader visual anxieties of the late-Renaissance period. To that extent, Chen-Morris's interest in cultural connections to visual paradoxes addresses similar issues as Stuart Clark's Vanities of the Eye, a book Chen-Morris rightly recognizes in his Introduction as "taking for granted the explanation of vision prevalent in that period" (p. 7). One might even approach Chen-Morris's book as focusing on similar concerns as Clark's, albeit with the eyes of a historian of science and a focus on Kepler in particular.

There is, however, one important aspect of Kepler's optics that Chen-Morris does not address. By the end of the book Chen-Morris has accomplished a helpful analysis of Kepler's optics and the way it addressed certain Copernican astronomical and philosophical questions, but it stops short of addressing the impact of Kepler's optics, or the issue of how his treatise actually affected Copernican reception. Such questions are unfortunately not considered in this 
book, which essentially focuses on Kepler's own ideas. Nevertheless, if the merit of a book is in part derived from the interesting questions that it raises, then one ought not fault Chen-Morris for making visible the intellectual development of Kepler's optical ideas. It will be for a different book to address whether, if at all, Kepler's optics refracted the visual culture of the time.

\author{
Brent Purkaple \\ University of Oklahoma \\ purkaple@ou.edu
}

